\title{
Introduction
}

\section{Current motor control issues in stroke rehabilitation}

\author{
Kathye E. Light* \\ Department of Physical Therapy, University of Florida Health and Science Center, Gainesville, FL 32610-0154, USA
}

The science of motor control provides dynamic and intriguing study for practical problems of concern to neurorehabilitation specialists. Two areas of present interest and controversy in stroke rehabilitation are addressed in this issue of NeuroRehabilitation. Section I of this journal will highlight current issues on the controversies of strengthening spastic muscle and forced-limb usage as therapeutic interventions.

Section II is devoted to the topic of motor control differences following right vs. left neurologic lesions. Both of these content areas represent current scientific debate in motor control science. I hope the readers of NeuroRehabilitation find this collection of scholarly works interesting and thought provoking.

To begin Section I: 'Strengthening and Forced-Limb Usage', Dr. Daniel Bourbonnais et al. present a case study demonstrating a particular type of strengthening model for motor reeducation. Based on the hypothesis that feedback on torque patterns will build strength and coordination for functional upper extremity control, Bour-

\footnotetext{
* Corresponding author. Tel.: +1 352 3950085; fax: + 1352 3926529.
}

bonnais et al. tested and discussed the feasibility and possible benefits of force modulation training with dynamometry. Gloria Miller and Kathye Light review the issue of strengthening as harmful to motor coordination and force control in clients with spastic hemiparesis. Ms. Miller and Dr. Light address a popular therapeutic treatment theory, and demonstrate the lack of research support for this 'clinical belief'. The last paper presented in Section I, 'Constraint-Induced Movement Therapy for Motor Recovery after Stroke' is an excellent paper explaining the details of the forced use approach studied by Taub et al. David Morris et al. reviews the history of Taub's research on constraint induction. After the research history and basis of the constraint induction model, Morris et al. describe the specific entrance criteria and methodology of the current protocal used by this research team for stroke rehabilitation. Clinicians and researchers alike will find this paper of practical importance.

Section II, addressing current issues on the different motor control effects of right vs. left brain lesions, opens with an engaging study on apraxia. Maher et al. studied clients with left and right strokes by videotaping gesture performance. 
Maher et al. explored the possibility that in addition to the left hemisphere, the right hemisphere may play a role in praxis performance. The second research study in Section II by Pohl et al. confirms the loss of sensory-motor control in the upper extremity ipsilateral to the lesion in addition to the involvement of the contralateral upper limb. These researchers emphasize the need for practice in speeded processing to improve the function of both upper limbs. The third study in Section II, by Giuliani et al. reveals the finding that subjects with left-sided stroke lesions had bilateral deficits in motor programming while those with right lesions had deficits only in the upper limb contralateral to the lesion. The authors discuss the significance of this finding to rehabilitation of clients post stroke. In the final article of Section II, Dr. Geralyn Schulz reviews the recent literature on speech production impairment following right and left hemisphere lesions. Dr. Schulz emphasizes the differences in impairment caused by not only right vs. left hemisphere damage, but also anterior vs. posterior lesions within these hemispheres. These differences have obvious implications for the rehabilitation of speech production for clients after stroke.

The researchers and authors of this edition join the guest editor, Dr. Kathye E. Light, in support of interdisciplinary research and scholarly review. We encourage the readers to discuss the controversies presented in this journal edition and to write us with points of interest or concern. 